\title{
Positive Data Visualization Using Trigonometric Function
}

\author{
Farheen Ibraheem, ${ }^{1}$ Maria Hussain, ${ }^{2}$ \\ Malik Zawwar Hussain, ${ }^{3}$ and Akhlaq Ahmad Bhatti ${ }^{1}$ \\ ${ }^{1}$ National University of Computer and Emerging Sciences, Lahore 54000, Pakistan \\ ${ }^{2}$ Lahore College for Women University, Lahore 54500, Pakistan \\ ${ }^{3}$ Department of Mathematics, University of the Punjab, Lahore 54590, Pakistan
}

Correspondence should be addressed to Farheen Ibraheem, farheen.butt@gmail.com

Received 6 June 2012; Revised 20 September 2012; Accepted 4 October 2012

Academic Editor: Kai Diethelm

Copyright ( 2012 Farheen Ibraheem et al. This is an open access article distributed under the Creative Commons Attribution License, which permits unrestricted use, distribution, and reproduction in any medium, provided the original work is properly cited.

A $C^{1}$ piecewise rational trigonometric cubic function with four shape parameters has been constructed to address the problem of visualizing positive data. Simple data-dependent constraints on shape parameters are derived to preserve positivity and assure smoothness. The method is then extended to positive surface data by rational trigonometric bicubic function. The order of approximation of developed interpolant is $O\left(h_{i}^{3}\right)$.

\section{Introduction}

Data visualization is the mechanism to communicate information by means of graphs, images, diagrams, and animations. It is extensively used in interactive simulation, geometrical design, geometric modeling, and computer-aided geometric design. It is an efficacious way to abridge complexity of data and facilitates prompt understanding of data.

The three significant features of data are convexity, monotonicity, and positivity. Either of these features arises in the data whether it is a result of physical process or chemical experiment, and so forth. Plenty of spline functions exist which can produce smooth and visibly pleasant curves but incapable to visualize the inherited shape (convexity, monotonicity, and positivity) of given data. In this paper, the positive data visualization of both curve and surface data is addressed by a rational trigonometric cubic function.

The objective of this paper is to preserve duly emphasized characteristic of data that is, positivity. Asim and Brodlie [1] developed a piecewise rational cubic function to preserve the positivity of positive data. In [1], if the interpolating function did not preserve the positivity in a subinterval, then the authors inserted extra knot to improve this matter. Butt 
and Brodlie [2] developed a piecewise rational cubic interpolant to preserve the shape of positive data by interval subdivision technique. Goodman et al. [3] constructed nonplanar shape preserving interpolating curve scheme. They obtained a curve through an optimization process involving some fairness criteria, in order to achieve curve by $G^{2}$ piecewise rational cubic function. Goodman [4] surveyed the shape preserving interpolating algorithms for 2D data. Han [5] presented the cubic trigonometric polynomial curves with shape parameters. It was investigated that partition of knot vector and value of free parameter affect the order of continuity of trigonometric polynomial curves with shape parameters. These cubic trigonometric polynomial curves were approximation of cubic B-spline curves but more convergent to control polygon. Their degree could be reduced to quadratic for a particular value of shape parameter.

Brodlie et al. [6] used piecewise bicubic function to preserve the shape of positive data arranged over the rectangular mesh. They developed sufficient conditions in the term of the first and mixed partial derivatives at the rectangular grid points to preserve positivity. Duan et al. [7] developed a bivariate rational interpolant with four shape parameters in each rectangular patch. The developed interpolant was $C^{1}$ for equally spaced data with a suitable choice of shape parameters. The sufficient restrictions were developed on shape parameters for constrained interpolation of data. M. Z. Hussain and M. Hussain [8] constructed a local positivity preserving scheme for positive data by making constraints on free parameters in the account of rational bicubic partially blended patches. In [8], the authors also developed the constraints on parameters to preserve the shape of data that is lying above the plane. The user did not enjoy the liberty to refine the curves and surface as desired in these schemes. Hussain and Sarfraz [9] developed a piecewise rational cubic function with four families of parameters to preserve the shape of positive data. Further, the authors extended a rational cubic function into rational bicubic function with eight free parameters for the data arranged over the rectangular grid. In [9], simple sufficient conditions were derived on these free parameters to preserve the shape of positive data. The scheme seemed to be computationally expansive.

Duan et al. [10] discussed the rate of convergence of a rational spline with two shape parameters. The range of optimal error coefficient was determined. The jump in the curvature was also studied for uniform data.

In this paper, an alternative cubic trigonometric function is used to preserve positivity of data. The developed scheme has ample useful aspects. It produces $C^{1}$ interpolant. No extra knots are inserted between any two knots to preserve positivity. The developed scheme works for both equally and unequally spaced data. Positivity is attained by imposing the data-dependent constraints on the free parameters rather than assuming certain functional values.

The work in this paper is set up in such a way that Section 2 elucidates the construction of the $C^{1}$ rational trigonometric cubic function to be used in curve scheme. Section 3 discusses the error of approximation. Section 4 extends the $C^{1}$ rational trigonometric cubic function to a $C^{1}$ rational trigonometric bicubic function. Constraints are developed on free parameters in Section 5 to preserve the positive shape of curve and surface data. Finally, Section 6 concludes the paper.

\section{2. $C^{1}$ Rational Trigonometric Cubic Function}

In this section, the $C^{1}$ piecewise rational trigonometric cubic function is developed. 
The curve data under consideration over the interval $[a, b]$ is $\left\{\left(x_{i}, y_{i}\right), i=0,1\right.$, $2, \ldots, n\}$, where the partition of data is $x_{0}<x_{1}<x_{2}<\cdots<x_{n}$. The rational trigonometric cubic function over each subinterval $I_{i}=\left[x_{i}, x_{i+1}\right]$ is defined as:

$$
S_{i}(x)=\frac{p_{i}(\theta)}{q_{i}(\theta)}
$$

with

$$
\begin{aligned}
p_{i}(\theta)= & \alpha_{i} f_{i}(1-\sin (\theta))^{3}+\left\{\beta_{i} f_{i}+\frac{2 h_{i} \alpha_{i} d_{i}}{\pi}\right\} \sin (\theta)(1-\sin (\theta))^{2} \\
& +\left\{\gamma_{i} f_{i+1}-\frac{2 h_{i} \delta_{i} d_{i+1}}{\pi}\right\} \cos (\theta)(1-\cos (\theta))^{2}+\delta_{i} f_{i+1}(1-\cos (\theta))^{3}, \\
q_{i}(\theta)= & \alpha_{i}(1-\sin (\theta))^{3}+\beta_{i} \sin (\theta)(1-\sin (\theta))^{2}+\gamma_{i} \cos (\theta)(1-\cos (\theta))^{2} \\
& +\delta_{i}(1-\cos (\theta))^{3}
\end{aligned}
$$

where

$$
\theta=\frac{\pi}{2}\left(\frac{x-x_{i}}{h_{i}}\right), \quad h_{i}=x_{i+1}-x_{i}
$$

The rational trigonometric cubic function (2.1) has the following properties:

$$
S\left(x_{i}\right)=f_{i}, \quad S\left(x_{i+1}\right)=f_{i+1}, \quad S^{(1)}\left(x_{i}\right)=d_{i}, \quad S^{(1)}\left(x_{i+1}\right)=d_{i+1} .
$$

Here, $S^{(1)}$ and $d_{i}$ are the derivatives with respect to $x$ and computed derivatives at knots $x_{i}$. The values of $d_{i}$ can be computed by any numerical scheme if not given with data. $S(x) \in C^{1}\left[x_{0}, x_{n}\right]$ has $\alpha_{i}$ and $\delta_{i}$ as free parameters.

\section{Error Estimation of Interpolation}

This section studies the approximation properties of rational trigonometric cubic function (2.1). It is assumed that the data is generated from third-order continuously differentiable function $f(x) \in C^{3}\left[x_{0}, x_{n}\right]$.Since the developed interpolation in Section 2 is local, the error of approximation is computed in the subinterval $\left[x_{i}, x_{i+1}\right]$. The absolute error is expressed in terms of Peono-Kernel [10] as follows:

$$
\left|f(x)-S_{i}(x)\right| \leq \frac{1}{2}\left\|f^{3}(x)\right\| \int_{x_{i}}^{x_{i+1}}\left|R_{x}\left[(x-\tau)_{+}^{2}\right]\right| d \tau,
$$


where $R_{x}\left[(x-\tau)_{+}^{2}\right]$ is the Peono-Kernel. $R_{x}\left[(x-\tau)_{+}^{2}\right]=r(\tau, x)$ for $x_{i}<\tau<x$ and $R_{x}\left[(x-\tau)_{+}^{2}\right]=s(\tau, x)$ for $x<\tau<x_{i+1}$. Therefore, the integral involved in (3.1) can be expressed as

$$
\int_{x_{i}}^{x_{i+1}}\left|R_{x}\left[(x-\tau)_{+}^{2}\right]\right| d \tau=\int_{x_{i}}^{x}|r(\tau, x)| d \tau+\int_{x}^{x_{i+1}}|s(\tau, x)| d \tau .
$$

For the $C^{1}$ rational trigonometric cubic function $(2.1), r(\tau, x)$ and $s(\tau, x)$ have the value

$$
\begin{gathered}
r(\tau, x)=(x-\tau)^{2}-\frac{1}{q_{i}(\theta)}\left\{\left(\gamma_{i} B_{2}+\delta_{i} B_{3}\right)\left(x_{i+1}-\tau\right)^{2}-\frac{4 h_{i} \delta_{i} B_{2}}{\pi}\left(x_{i+1}-\tau\right)\right\}, \\
s(\tau, x)=-\frac{1}{q_{i}(\theta)}\left\{\left(\gamma_{i} B_{2}+\delta_{i} B_{3}\right)\left(x_{i+1}-\tau\right)^{2}-\frac{4 h_{i} \delta_{i} B_{2}}{\pi}\left(x_{i+1}-\tau\right)\right\} \\
B_{2}=\cos (\theta)(1-\cos (\theta))^{2}, \quad B_{3}=(1-\cos (\theta))^{3} .
\end{gathered}
$$

To compute the integral of absolute values in (3.1), the roots of $r(\tau, x)$ and $s(\tau, x)$ are calculated. The roots of $r(x, x)$ in $[0,1]$ are $\theta=1, \theta^{*}=1-2 \delta_{i} / \pi\left(\gamma_{i}-\delta_{i}\right)$.

The roots of $r(\tau, x)=0$ are $\tau_{1}=x+h_{i}((B-D) / A)$ and $\tau_{2}=x+h_{i}((B+D) / A)$, where

$$
\begin{gathered}
A=2\left(\alpha_{i} B_{0}+\beta_{i} B_{1}\right), \quad B=\frac{4 B_{2} \delta_{i}-2 \pi\left(B_{2} \gamma_{i}+B_{3} \delta_{i}\right)(1-\theta)}{\pi}, \\
D=\sqrt{\sigma-\frac{4\left(\alpha_{i} B_{0}+\beta_{i} B_{1}\right)\left(4 B_{2} \delta_{i}(1-\theta)-\pi\left(B_{2} \gamma_{i}+B_{3} \delta_{i}\right)(1-\theta)^{2}\right)}{\pi}}, \\
\sigma=\left[\frac{4 B_{2} \delta_{i}-2 \pi\left(B_{2} \gamma_{i}+B_{3} \delta_{i}\right)(1-\theta)}{\pi}\right]^{2} \quad B_{0}=(1-\sin (\theta))^{3}, \quad B_{1}=\sin (\theta)(1-\sin (\theta))^{2} .
\end{gathered}
$$

$\sigma$ denotes $\left[\left(4 B_{2} \delta_{i}-2 \pi\left(B_{2} \gamma_{i}+B_{3} \delta_{i}\right)(1-\theta)\right) / \pi\right]^{2}$.

The roots of $s(\tau, x)$ are $\tau_{3=x_{i+1}}$ and $\tau_{4}=x_{i+1}-4 h_{i} \delta_{i} B_{2} / \pi\left(\gamma_{i} B_{2}-\delta_{i} B_{3}\right)$. 
The following possible cases arise.

Case 1. $0 \leq \gamma_{i} \leq 1,0 \leq \theta \leq 1$, (3.1) takes the form

$$
\begin{gathered}
\left|f(x)-S_{i}(x)\right| \leq \frac{1}{2}\left\|f^{3}(\tau)\right\| h_{i}^{3} \omega_{1}\left(\delta_{i}, \gamma_{i}, \theta\right) \\
\omega_{1}\left(\delta_{i}, \gamma_{i}, \theta\right)=\int_{x_{i}}^{x}|r(\tau, x)| d \tau+\int_{x}^{x_{i+1}}|s(\tau, x)| d \tau \\
=\frac{1}{q_{i}(\theta)}\left\{\frac{2 \gamma_{i} B_{2} \theta^{2}-\pi(1-\theta)\left(\gamma_{i} B_{2}+\delta_{i} B_{3}\right) \theta^{2}}{\pi}\right. \\
+\frac{4 \gamma_{i} B_{2}(1-\theta) \theta-\pi\left(\gamma_{i} B_{2}+\delta_{i} B_{3}\right)(1-\theta)^{2} \theta}{\pi} \\
\left.+\frac{\left(\alpha_{i} B_{0}+\beta_{i} B_{1}\right) \theta^{3}}{3}+\frac{(1-\theta)^{2}\left(6 \gamma_{i} B_{2}-\pi\left(\gamma_{i} B_{2}+\delta_{i} B_{3}\right)(1-\theta)\right)}{3 \pi}\right\} .
\end{gathered}
$$

Case 2. $\gamma_{i}>1+2 / \pi \delta_{i}, 0 \leq \theta \leq \theta^{*}$, (3.1) takes the form

$$
\begin{aligned}
&\left|f(x)-S_{i}(x)\right| \leq \frac{1}{2}\left\|f^{3}(\tau)\right\| h_{i}^{3} \omega_{2}\left(\delta_{i}, \gamma_{i}, \theta\right), \\
& \omega_{2}\left(\delta_{i}, \gamma_{i}, \theta\right)=\int_{x_{i}}^{x}|r(\tau, x)| d \tau+\int_{x}^{x_{i+1}}|s(\tau, x)| d \tau \\
&=\frac{1}{q_{i}(\theta)}\{- \frac{2\left(\alpha_{i} B_{0}+\beta_{i} B_{1}\right)}{3}\left(\frac{B-D}{A}\right)^{3} \\
&+\frac{\left(4 \delta_{i} B_{2}-2 \pi(1-\theta)\left(\gamma_{i} B_{2}+B_{3}\right)\right)}{\pi}\left(\frac{B-D}{A}\right)^{2} \\
&-\left(\frac{8 \delta_{i} B_{2}(1-\theta)-2 \pi\left(\gamma_{i} B_{2}+\delta_{i} B_{3}\right)(1-\theta)^{2}}{\pi}\right)\left(\frac{B-D}{A}\right) \\
&\left.-\frac{\left(\alpha_{i} B_{0}+\beta_{i} B_{1}\right) \theta^{3}-\left(\frac{2 \delta_{i} B_{2}-(1-\theta)\left(\gamma_{i} B_{2}+\delta_{i} B_{3}\right)}{3}\right) \theta^{2}}{\pi}\right) \theta+\frac{64 \delta_{i} B_{2}}{3 \pi^{3}\left(\gamma_{i} B_{2}+\delta_{i} B_{3}\right)^{2}} \\
&-\left(\frac{4 \delta_{i} B_{2}(1-\theta)-\left(\gamma_{i} B_{2}+\delta_{i} B_{3}\right)(1-\theta)^{2}}{\pi}\right) \\
&\left.-\frac{2 \delta_{i} B_{2}(1-\theta)^{2}}{\pi}+\frac{(1-\theta)^{3}\left(\gamma_{i} B_{2}+\delta_{i} B_{3}\right)}{3}\right\} .
\end{aligned}
$$

Case 3. $\theta^{*} \leq \theta \leq 1$, (3.1) takes the form

$$
\left|f(x)-S_{i}(x)\right| \leq \frac{1}{2}\left\|f^{3}(\tau)\right\| h_{i}^{3} \omega_{3}\left(\delta_{i}, \gamma_{i}, \theta\right),
$$


where

$$
\begin{aligned}
& \omega_{3}\left(\delta_{i}, \gamma_{i}, \theta\right)=\int_{x_{i}}^{x}|r(\tau, x)| d \tau+\int_{x}^{x_{i+1}}|s(\tau, x)| d \tau \\
&=\frac{1}{q_{i}(\theta)}\left\{\frac{2\left(\alpha_{\mathrm{i}} B_{0}+\beta_{\mathrm{i}} B_{1}\right)}{3}\left(\frac{B-D}{A}\right)^{3}\right. \\
&+ \frac{\left(4 \delta_{i} B_{2}-2 \pi(1-\theta)\left(\gamma_{\mathrm{i}} B_{2}+B_{3}\right)\right)}{\pi}\left(\frac{B-D}{A}\right)^{2} \\
&+\left(\frac{8 \delta_{i} B_{2}(1-\theta)-2 \pi\left(\gamma_{i} B_{2}+\delta_{i} B_{3}\right)(1-\theta)^{2}}{\pi}\right)\left(\frac{B-D}{A}\right) \\
&\left.-\frac{2\left(\alpha_{i} B_{0}+\beta_{i} B_{1}\right)}{3}\left(\frac{B+D}{A}\right)^{3}\right)\left(\frac{B+D}{A}\right)^{2}+\frac{\left(\alpha_{\mathrm{i}} B_{0}+\beta_{\mathrm{i}} B_{1}\right) \theta^{3}}{3} \\
&+\left(\frac{4 \delta_{i} B_{2}-2 \pi(1-\theta)\left(\gamma_{i} B_{2}+\delta_{i} B_{3}\right)}{\pi}\right)\left(\frac{B+D}{A}\right) \\
&\left.+\left(\frac{8 \delta_{i} B_{2}(1-\theta)-2 \pi\left(\gamma_{i} B_{2}+\delta_{i} B_{3}\right)(1-\theta)^{2}}{\pi}\right)(1-\theta)^{2}\right\} . \\
&+\left(\frac{2 \delta_{i} B_{2}-\pi(1-\theta)\left(\gamma_{i} B_{2}+\delta_{i} B_{3}\right)}{\pi}\right) \theta^{2} \\
&+\left(\frac{4 \delta_{i} B_{2}(1-\theta)-\pi(1-\theta)^{2}\left(\gamma_{i} B_{2}+\delta_{i} B_{3}\right)}{\pi}\right) \\
&\left(\frac{6 \delta_{i} B_{2}-\pi\left(\gamma_{i} B_{2}+\delta_{i} B_{3}\right)(1-\theta)}{3 \pi}\right)
\end{aligned}
$$

The above can be summarized as follows.

Theorem 3.1. The error of $C^{1}$ rational trigonometric cubic function (2.1), for $f(x) \in C^{3}\left[x_{0}, x_{n}\right]$, in each subinterval $\left[x_{i}, x_{i+1}\right]$ is

$$
\begin{gathered}
\left|f(x)-S_{i}(x)\right| \leq \frac{1}{2}\left\|f^{3}(x)\right\| h_{i}^{3} c_{i}, \\
c_{i}=\max _{0 \leq \theta \leq 1} \omega\left(\delta_{i}, \gamma_{i}, \theta\right), \\
\omega\left(\delta_{i}, \gamma_{i}, \theta\right)= \begin{cases}\max \omega_{1}\left(\delta_{i}, \gamma_{i}, \theta\right), & 0 \leq \gamma_{i} \leq 1,0 \leq \theta \leq 1, \\
\max \omega_{2}\left(\delta_{i}, \gamma_{i}, \theta\right), & \gamma_{i}>1+\frac{2}{\pi} \delta_{i}, 0 \leq \theta \leq \theta^{*}, \\
\max \omega_{3}\left(\delta_{i}, \gamma_{i}, \theta\right), & \theta^{*} \leq \theta \leq 1 .\end{cases}
\end{gathered}
$$




\section{Rational Trigonometric Bicubic Function}

The rational trigonometric cubic function (2.1) is extended to a rational bicubic function defined over a rectangular mesh $D=\left[x_{0}, x_{m}\right] \times\left[y_{0}, y_{n}\right]$. Let $d: a=x_{0}<x_{1}<\cdots<x_{m}=b$ be a partition of $[a, b]$ and $d_{1}: c=y_{0}<y_{1}<\cdots<y_{n}=d$ be a partition of $[c, d]$. Rational trigonometric bicubic function is defined over each rectangular patch $\left[x_{i}, x_{i+1}\right] \times\left[y_{j}, y_{j+1}\right], i=$ $0,1, \ldots, m-1 ; j=0,1, \ldots, n-1$ as follows:

$$
S(x, y)=S_{i, j}(x, y)=A_{i}(\theta) F(i, j) \widehat{A}_{j}^{T}(\varphi),
$$

where

$$
\begin{aligned}
& F(i, j)=\left[\begin{array}{cccc}
F_{i, j} & F_{i, j+1} & F_{i, j}^{y} & F_{i, j+1}^{y} \\
F_{i+1, j} & F_{i+1, j+1} & F_{i+1, j}^{y} & F_{i+1, j+1}^{y} \\
F_{i, j}^{x} & F_{i, j+1}^{x} & F_{i, j}^{x y} & F_{i, j+1}^{x y} \\
F_{i+1, j}^{x} & F_{i+1, j+1}^{x} & F_{i+1, j}^{x y} & F_{i+1, j+1}^{x y}
\end{array}\right],
\end{aligned}
$$

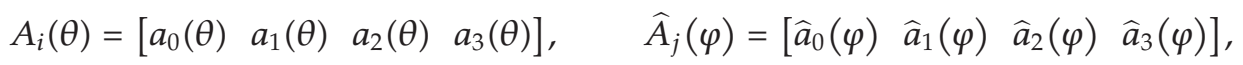

$$
\begin{aligned}
& a_{0}(\theta)=\frac{\alpha_{i, j}(1-\sin (\theta))^{3}+\beta_{i, j} \sin (\theta)(1-\sin (\theta))^{2}}{q_{i}(\theta)}, \\
& a_{1}(\theta)=\frac{\gamma_{i, j} \cos (\theta)(1-\cos (\theta))^{2}+\delta_{i, j}(1-\cos (\theta))^{3}}{q_{i}(\theta)}, \\
& a_{2}(\theta)=\frac{2 h_{i} \alpha_{i, j} \sin (\theta)(1-\sin (\theta))^{2}}{\pi q_{i}(\theta)}, \quad a_{3}(\theta)=\frac{-2 h_{i} \delta_{i, j} \cos (\theta)(1-\cos (\theta))^{2}}{\pi q_{i}(\theta)}, \\
& q_{i}(\theta)=\alpha_{i, j}(1-\sin (\theta))^{3}+\beta_{i, j} \sin (\theta)(1-\sin (\theta))^{2} \\
& +\gamma_{i, j} \cos (\theta)(1-\cos (\theta))^{2}+\delta_{i, j}(1-\cos (\theta))^{3}, \\
& \widehat{a}_{0}(\varphi)=\frac{\widehat{\alpha}_{i, j}(1-\sin (\varphi))^{3}+\widehat{\beta}_{i, j} \sin (\varphi)(1-\sin (\varphi))^{2}}{q_{j}(\varphi)}, \\
& \widehat{a}_{1}(\varphi)=\frac{\widehat{\gamma}_{i, j} \cos (\varphi)(1-\cos (\varphi))^{2}+\widehat{\delta}_{i, j}(1-\cos (\varphi))^{3}}{q_{j}(\varphi)}, \\
& \widehat{a}_{2}(\varphi)=\frac{2 h_{j} \widehat{\alpha}_{i, j} \sin (\varphi)(1-\sin (\varphi))^{2}}{\pi q_{j}(\varphi)}, \quad \widehat{a}_{3}(\varphi)=\frac{-2 h_{j} \widehat{\delta}_{i, j} \cos (\varphi)(1-\cos (\varphi))^{2}}{\pi q_{j}(\varphi)}, \\
& q_{j}(\varphi)=\widehat{\alpha}_{i, j}(1-\sin (\varphi))^{3}+\widehat{\beta}_{i, j} \sin (\varphi)(1-\sin (\varphi))^{2} \\
& +\widehat{\gamma}_{i, j} \cos (\varphi)(1-\cos (\varphi))^{2}+\widehat{\delta}_{i, j}(1-\cos (\varphi))^{3}, \\
& \varphi=\frac{\pi}{2}\left(\frac{y-y_{j}}{h_{j}}\right) .
\end{aligned}
$$

The entries of $F(i, j)$ are the first and mixed partial derivatives at the four corner positions of the cubic patch. 


\section{1. $C^{1}$ Rational Trigonometric Bicubic Function}

The rational trigonometric function defined in (3.2) interpolates the data values $F_{i, j}$ and partial derivatives $F_{i, j}^{x}, F_{i, j}^{y}, F_{i, j}^{x y}$ defined at four corners of rectangular patch, that is,

$$
S\left(x_{i}, y_{j}\right)=F_{i, j}, \quad \frac{\partial S\left(x_{i}, y_{j}\right)}{\partial x}=F_{i, j^{\prime}}^{x}, \quad \frac{\partial S\left(x_{i}, y_{j}\right)}{\partial y}=F_{i, j^{\prime}}^{y} \quad \frac{\partial S\left(x_{i}, y_{j}\right)}{\partial x \partial y}=F_{i, j}^{x y} .
$$

Since each rectangular patch is bounded by four boundary curves so to blend the rectangular patches to generate a $C^{1}$ continuous surface, following sufficient conditions must be satisfied along the four boundaries of each rectangular patch:

$$
\begin{gathered}
\left.\frac{\partial S_{i, j}\left(x_{i+1}, y\right)}{\partial x}\right|_{\theta=\pi / 2}-\left.\frac{\partial S_{i+1, j}\left(x_{i+1}, y\right)}{\partial x}\right|_{\theta=0}=0, \\
\left.\frac{\partial S_{i-1, j}\left(x_{i}, y\right)}{\partial x}\right|_{\theta=\pi / 2}-\left.\frac{\partial S_{i, j}\left(x_{i}, y\right)}{\partial x}\right|_{\theta=0}=0, \\
\left.\frac{\partial S_{i, j}\left(x, y_{j+1}\right)}{\partial y}\right|_{\varphi=\pi / 2}-\left.\frac{\partial S_{i, j+1}\left(x, y_{j+1}\right)}{\partial y}\right|_{\varphi=0}=0, \\
\left.\frac{\partial S_{i, j-1}\left(x, y_{j}\right)}{\partial y}\right|_{\varphi=\pi / 2}-\left.\frac{\partial S_{i, j}\left(x, y_{j}\right)}{\partial y}\right|_{\varphi=0}=0 .
\end{gathered}
$$

The entities $h_{i}, h_{j}, F_{i, j}, F_{i, j}^{x}, F_{i, j}^{y}, F_{i, j}^{x y}, i=0,1,2, \ldots, m ; j=0,1,2, \ldots, n$ are assumed fixed then, we have the following observations: $\partial S_{i, j}\left(x_{i+1}, y\right) /\left.\partial x\right|_{\theta=\pi / 2}-\left(\partial S_{i+1, j}\left(x_{i+1}, y\right)\right) /\left.\partial x\right|_{\theta=0}=0$ if

$$
\begin{gathered}
\pi h_{i} \alpha_{i+1, j} \beta_{i+1, j} \delta_{i, j}^{2} F_{i+1, j}\left(\widehat{\alpha}_{i, j}-\widehat{\alpha}_{i+1, j}\right)=0, \\
2 \pi h_{i} h_{i+1} \alpha_{i+1, j}^{2} \delta_{i, j}^{2} F_{i+1, j}^{x}\left(\widehat{\beta}_{i, j}-\widehat{\beta}_{i+1, j}\right)+4 h_{i} h_{i+1} h_{j} \alpha_{i+1, j}^{2} \delta_{i, j}^{2} F_{i+1, j}^{x y}\left(\widehat{\alpha}_{i, j}-\widehat{\alpha}_{i+1, j}\right)=0, \\
2 \pi h_{i} h_{i+1} \alpha_{i+1, j}^{2} \delta_{i, j}^{2} F_{i+1, j+1}^{x}\left(\widehat{\gamma}_{i, j}-\widehat{\gamma}_{i+1, j}\right)+4 h_{i} h_{i+1} h_{j} \alpha_{i+1, j}^{2} \delta_{i, j}^{2} F_{i+1, j+1}^{x y}\left(\widehat{\delta}_{i, j}-\widehat{\delta}_{i+1, j}\right)=0, \\
2 \alpha_{i+1, j}^{2} h_{i} h_{i+1} \delta_{i, j}^{2} F_{i+1, j+1}^{x}\left(\widehat{\delta}_{i, j}-\widehat{\delta}_{i+1, j}\right)=0,
\end{gathered}
$$

then

(1) $\pi h_{i} \alpha_{i+1, j} \beta_{i+1, j} \delta_{i, j}^{2} F_{i+1, j}\left(\widehat{\alpha}_{i, j}-\widehat{\alpha}_{i+1, j}\right)=0$ if $\widehat{\alpha}_{i, j}=\widehat{\alpha}_{i+1, j}$,

(2) $2 \pi h_{i} h_{i+1} \alpha_{i+1, j}^{2} \delta_{i, j}^{2} F_{i+1, j}^{x}\left(\widehat{\beta}_{i, j}-\widehat{\beta}_{i+1, j}\right)+4 h_{i} h_{i+1} h_{j} \alpha_{i+1, j}^{2} \delta_{i, j}^{2} F_{i+1, j}^{x y}\left(\widehat{\alpha}_{i, j}-\widehat{\alpha}_{i+1, j}\right)=0$ if $\widehat{\alpha}_{i, j}=\widehat{\alpha}_{i+1, j}$, $\widehat{\beta}_{i, j}=\widehat{\beta}_{i+1, j}$

(3) $2 \pi h_{i} h_{i+1} \alpha_{i+1, j}^{2} \delta_{i, j}^{2} F_{i+1, j+1}^{x}\left(\widehat{\gamma}_{i, j}-\widehat{\gamma}_{i+1, j}\right)+4 h_{i} h_{i+1} h_{j} \alpha_{i+1, j}^{2} \delta_{i, j}^{2} F_{i+1, j+1}^{x y}\left(\widehat{\delta}_{i, j}-\widehat{\delta}_{i+1, j}\right)=0$ if $\widehat{\gamma}_{i, j}=$ $\widehat{\gamma}_{i+1, j}, \widehat{\delta}_{i, j}=\widehat{\delta}_{i+1, j}$, 
Journal of Applied Mathematics

(4) $2 \alpha_{i+1, j}^{2} h_{i} h_{i+1} \delta_{i, j}^{2} F_{i+1, j+1}^{x}\left(\widehat{\delta}_{i, j}-\widehat{\delta}_{i+1, j}\right)=0$ if $\widehat{\delta}_{i, j}=\widehat{\delta}_{i+1, j}$. Consider that

$$
\left.\frac{\partial S_{i-1, j}\left(x_{i}, y\right)}{\partial x}\right|_{\theta=\pi / 2}-\left.\frac{\partial S_{i, j}\left(x_{i}, y\right)}{\partial x}\right|_{\theta=0}=0
$$

if

$$
\begin{gathered}
2 \alpha_{i, j}^{2} h_{i} h_{i-1} \delta_{i-1, j}^{2} F_{i, j}^{x}\left(\widehat{\alpha}_{i-1, j}-\widehat{\alpha}_{i, j}\right)=0, \\
2 \pi \alpha_{i, j}^{2} h_{i} h_{i-1} \delta_{i-1, j}^{2} F_{i, j}^{x}\left(\widehat{\beta}_{i-1, j}-\widehat{\beta}_{i, j}\right)+4 \alpha_{i, j}^{2} h_{i} h_{i-1} \delta_{i-1, j}^{2} F_{i, j}^{x}\left(\widehat{\alpha}_{i-1, j}-\widehat{\alpha}_{i, j}\right)=0, \\
4 \alpha_{i, j}^{2} h_{i} h_{i-1} \delta_{i-1, j}^{2} F_{i, j+1}^{x y}\left(\widehat{\delta}_{i-1, j}-\widehat{\delta}_{i, j}\right)+2 \pi \alpha_{i, j}^{2} h_{i} h_{i-1} \delta_{i-1, j}^{2} F_{i, j}^{x}\left(\widehat{\gamma}_{i-1, j}-\widehat{\gamma}_{i, j}\right)=0, \\
2 \alpha_{i, j}^{2} h_{i} h_{i-1} \delta_{i-1, j}^{2} F_{i, j+1}^{x}\left(\widehat{\delta}_{i-1, j}-\widehat{\delta}_{i, j}\right)=0,
\end{gathered}
$$

then

(1) $2 \alpha_{i, j}^{2} h_{i} h_{i-1} \delta_{i-1, j}^{2} F_{i, j}^{x}\left(\widehat{\alpha}_{i-1, j}-\widehat{\alpha}_{i, j}\right)=0$ if $\widehat{\alpha}_{i-1, j}=\widehat{\alpha}_{i, j}$,

(2) $2 \pi \alpha_{i, j}^{2} h_{i} h_{i-1} \delta_{i-1, j}^{2} F_{i, j}^{x}\left(\widehat{\beta}_{i-1, j}-\widehat{\beta}_{i, j}\right)+4 \alpha_{i, j}^{2} h_{i} h_{i-1} \delta_{i-1, j}^{2} F_{i, j}^{x}\left(\widehat{\alpha}_{i-1, j}-\widehat{\alpha}_{i, j}\right)=0$ if $\widehat{\beta}_{i-1, j}=\widehat{\beta}_{i, j}$ and $\widehat{\alpha}_{i-1, j}=\widehat{\alpha}_{i, j}$,

(3) $4 \alpha_{i, j}^{2} h_{i} h_{i-1} \delta_{i-1, j}^{2} F_{i, j+1}^{x y}\left(\widehat{\delta}_{i-1, j}-\widehat{\delta}_{i, j}\right)+2 \pi \alpha_{i, j}^{2} h_{i} h_{i-1} \delta_{i-1, j}^{2} F_{i, j}^{x}\left(\widehat{\gamma}_{i-1, j}-\widehat{\gamma}_{i, j}\right)=0$ if $\widehat{\delta}_{i-1, j}=\widehat{\delta}_{i, j}$ and $\widehat{\gamma}_{i-1, j}=\widehat{\gamma}_{i, j}$,

(4) $2 \alpha_{i, j}^{2} h_{i} h_{i-1} \delta_{i-1, j}^{2} F_{i, j+1}^{x}\left(\widehat{\delta}_{i-1, j}-\widehat{\delta}_{i, j}\right)=0$ if $\widehat{\delta}_{i-1, j}=\widehat{\delta}_{i, j}$. Consider that

$$
\left.\frac{\partial S_{i, j}\left(x, y_{j+1}\right)}{\partial y}\right|_{\varphi=\pi / 2}-\left.\frac{\partial S_{i, j+1}\left(x, y_{j+1}\right)}{\partial y}\right|_{\varphi=0}=0
$$

if

$$
\begin{gathered}
\widehat{\alpha}_{i, j+1}^{2} \widehat{\delta}_{i, j}^{2} F_{i, j+1}^{y}\left(\alpha_{i, j}-\alpha_{i, j+1}\right)=0, \\
2 h_{i} \widehat{\delta}_{i, j}^{2} F_{i, j+1}^{x y}\left(\alpha_{i, j}-\alpha_{i, j+1}\right)=0, \\
\pi \widehat{\alpha}_{i, j+1}^{2} \widehat{\delta}_{i, j}^{2} F_{i+1, j+1}^{y}\left(\gamma_{i, j}-\gamma_{i, j+1}\right)+2 h_{i} \widehat{\alpha}_{i, j+1}^{2} \widehat{\delta}_{i, j}^{2} F_{i+1, j+1}^{x y}\left(\delta_{i, j}-\delta_{i, j+1}\right)=0, \\
\widehat{\delta}_{i, j}^{2} \widehat{\alpha}_{i, j+1}^{2} F_{i+1, j+1}^{y}\left(\delta_{i, j}-\delta_{i, j+1}\right)=0,
\end{gathered}
$$

then

(1) $\hat{\alpha}_{i, j+1}^{2} \widehat{\delta}_{i, j}^{2} F_{i, j+1}^{y}\left(\alpha_{i, j}-\alpha_{i, j+1}\right)=0$ if $\alpha_{i, j}=\alpha_{i, j+1}$,

(2) $2 h_{i} \widehat{\delta}_{i, j}^{2} F_{i, j+1}^{x y}\left(\alpha_{i, j}-\alpha_{i, j+1}\right)=0$ if $\alpha_{i, j}=\alpha_{i, j+1}$, 
(3) $\pi \widehat{\alpha}_{i, j+1}^{2} \widehat{\delta}_{i, j}^{2} F_{i+1, j+1}^{y}\left(\gamma_{i, j}-\gamma_{i, j+1}\right)+2 h_{i} \widehat{\alpha}_{i, j+1}^{2} \widehat{\delta}_{i, j}^{2} F_{i+1, j+1}^{x y}\left(\delta_{i, j}-\delta_{i, j+1}\right)=0$ if $\gamma_{i, j}=\gamma_{i, j+1}, \delta_{i, j}=$ $\delta_{i, j+1}$

(4) $\widehat{\delta}_{i, j}^{2} \widehat{\widehat{\alpha}}_{i, j+1}^{2} F_{i+1, j+1}^{y}\left(\delta_{i, j}-\delta_{i, j+1}\right)=0$ if $\delta_{i, j}=\delta_{i, j+1}$. Consider that

$$
\left.\frac{\partial S_{i, j-1}\left(x, y_{j}\right)}{\partial y}\right|_{\varphi=\pi / 2}-\left.\frac{\partial S_{i, j}\left(x, y_{j}\right)}{\partial y}\right|_{\varphi=0}=0
$$

if

$$
\begin{gathered}
\widehat{\alpha}_{i, j}^{2} \widehat{\delta}_{i, j-1}^{2} F_{i, j}^{y}\left(\alpha_{i, j-1}-\alpha_{i, j}\right)=0, \\
\pi \widehat{\alpha}_{i, j}^{2} \widehat{\delta}_{i, j-1}^{2} F_{i, j}^{y}\left(\beta_{i, j-1}-\beta_{i, j}\right)+2 h_{i} \widehat{\alpha}_{i, j}^{2} \widehat{\delta}_{i, j-1}^{2} F_{i, j}^{x y}\left(\alpha_{i, j-1}-\alpha_{i, j}\right)=0, \\
\pi \widehat{\alpha}_{i, j}^{2} \widehat{\delta}_{i, j-1}^{2} F_{i+1, j}^{y}\left(\gamma_{i, j-1}-\gamma_{i, j}\right)+2 h_{i} \widehat{\alpha}_{i, j}^{2} \widehat{\delta}_{i, j-1}^{2} F_{i, j}^{x y}\left(\delta_{i, j-1}-\delta_{i, j}\right)=0, \\
\widehat{\alpha}_{i, j}^{2} \widehat{\delta}_{i, j-1}^{2} F_{i+1, j}^{y}\left(\delta_{i, j-1}-\delta_{i, j}\right)=0,
\end{gathered}
$$

then

(1) $\widehat{\alpha}_{i, j}^{2} \widehat{\delta}_{i, j-1}^{2} F_{i, j}^{y}\left(\alpha_{i, j-1}-\alpha_{i, j}\right)=0$ if $\alpha_{i, j-1}=\alpha_{i, j}$,

(2) $\pi \widehat{\alpha}_{i, j}^{2} \widehat{\delta}_{i, j-1}^{2} F_{i, j}^{y}\left(\beta_{i, j-1}-\beta_{i, j}\right)+2 h_{i} \widehat{\alpha}_{i, j}^{2} \widehat{\delta}_{i, j-1}^{2} F_{i, j}^{x y}\left(\alpha_{i, j-1}-\alpha_{i, j}\right)=0$ if $\beta_{i, j-1}=\beta_{i, j}$ and $\alpha_{i, j-1}=\alpha_{i, j}$,

(3) $\pi \widehat{\alpha}_{i, j}^{2} \widehat{\delta}_{i, j-1}^{2} F_{i+1, j}^{y}\left(\gamma_{i, j-1}-\gamma_{i, j}\right)+2 h_{i} \widehat{\alpha}_{i, j}^{2} \widehat{\delta}_{i, j-1}^{2} F_{i, j}^{x y}\left(\delta_{i, j-1}-\delta_{i, j}\right)=0$ if $\gamma_{i, j-1}=\gamma_{i, j}$ and $\delta_{i, j-1}=\delta_{i, j}$,

(4) $\widehat{\alpha}_{i, j}^{2} \widehat{\delta}_{i, j-1}^{2} F_{i+1, j}^{y}\left(\delta_{i, j-1}-\delta_{i, j}\right)=0$ if $\delta_{i, j-1}=\delta_{i, j}$.

The above discussion is summarized as follows.

Theorem 4.1. The piecewise bivariate rational cubic trigonometric function $S(x, y)$ defined in (4.1) is $C^{1}$ over the whole domain if the shape design parameters satisfy the following relations:

(1) $\alpha_{i, j}=\alpha_{i}, \beta_{i, j}=\beta_{i}, \gamma_{i, j}=\gamma_{i}, \delta_{i, j}=\delta_{i}, i=0,1,2, \ldots, m-1$ and for all values of $j$.

(2) $\widehat{\alpha}_{i, j}=\widehat{\alpha}_{j}, \widehat{\beta}_{i, j}=\widehat{\beta}_{j}, \widehat{\gamma}_{i, j}=\widehat{\gamma}_{j}, \widehat{\delta}_{i, j}=\widehat{\delta}_{j}, j=0,1,2, \ldots, n-1$ and for all values of $i$.

\section{Positivity Preserving Techniques}

Preserving positivity is the essence of data visualization in many fields of study. There are many physical situations where the entities only have meaning when their values are positive. For instance, population density, probability distribution, amount of rain fall, and resistance of an electric circuit are some of the areas where data values can not be negative. Similarly, terrain modeling, formation of geological crust movement to predict earth quake and volcanic eruptions, fluid dynamic, and carbon dating are few of the fields in which the resulting surface is required to be positive. Therefore, it is customary that interpolating curve and surface inherent the positivity of data. The subsequent subsections aim at developing constraints on the shape parameters in the description of rational trigonometric function and rational trigonometric bicubic function so that the resultant curve and surface are positive for a positive data and visibly eye catching. 


\subsection{Positive Curve Interpolation}

The positive curve data is data set $\left\{\left(x_{i}, f_{i}\right), i=0,1,2, \ldots, n\right\}$ satisfying the condition

$$
f_{i}>0, \quad i=0,1,2, \ldots, n-1 \text {. }
$$

The piecewise rational trigonometric cubic function defined in (2.1) inherits the positive shape in curve if in each subinterval $I_{i}=\left[x_{i}, x_{i+1}\right]$ the following relation is true

$$
S_{i}(x)>0, \quad i=0,1,2, \ldots, n-1 .
$$

The central idea is to impose conditions on free parameters to assure positivity. It can be observed that strictly positive denomination $q_{i}(\theta)$ can be guaranteed if

$$
\alpha_{i}>0, \quad \beta_{i}>0, \quad \gamma_{i}>0, \quad \delta_{i}>0
$$

Thus, the positivity of $S_{i}(x)=p_{i}(\theta) / q_{i}(\theta)$ depends on the positivity of the trigonometric cubic polynomial $p_{i}(\theta)$, and the difficulty level only reduces to the determination of suitable values of $\alpha_{i}, \beta_{i}, \gamma_{i}$ and $\delta_{i}$; for which the polynomial $p_{i}(\theta)>0$.

$p_{i}(\theta)>0$ if the coefficients $A_{i}, i=0,1,2,3$, of trigonometric basis function are positive. Positivity of these coefficients yields the following result:

$$
\beta_{i}>\frac{-2 h_{i} d_{i} \alpha_{i}}{\pi f_{i}}, \quad \gamma_{i}>\frac{2 h_{i} d_{i+1} \delta_{i}}{\pi f_{i+1}}
$$

The above discussion can be summarized as follows.

Theorem 5.1. The $C^{1}$ piecewise rational trigonometric cubic function preserve the positivity of positive data if in each subinterval $I_{i}=\left[x_{i}, x_{i+1}\right]$, the parameters $\beta_{i}$ and $\gamma_{i}$ satisfy the following sufficient conditions:

$$
\begin{aligned}
& \beta_{i}>\operatorname{Max}\left\{0, \frac{-2 h_{i} d_{i} \alpha_{i}}{\pi f_{i}}\right\}, \\
& \gamma_{i}>\operatorname{Max}\left\{0, \frac{2 h_{i} d_{i+1} \delta_{i}}{\pi f_{i+1}}\right\} .
\end{aligned}
$$

The above constraints can be rearranged as follows:

$$
\begin{array}{ll}
\beta_{i}=u_{i}+\operatorname{Max}\left\{0, \frac{-2 h_{i} d_{i} \alpha_{i}}{\pi f_{i}}\right\}, & u_{i}>0, \\
\gamma_{i}=v_{i}+\operatorname{Max}\left\{0, \frac{2 h_{i} d_{i+1} \delta_{i}}{\pi f_{i+1}}\right\}, & v_{i}>0 .
\end{array}
$$

Proof. The Theorem 5.1 can be easily established by combining (5.3) and (5.4). 


\section{2. $C^{1}$ Positive Surface Interpolation}

Let $\left\{F_{i, j}: i=0,1,2, \ldots, m ; j=0,1,2, \ldots, n\right\}$ be the positive data, that is, $F_{i, j}>0, i=$ $0,1,2, \ldots, m ; j=0,1,2, \ldots, n$. The aim is to construct a $C^{1}$ rational trigonometric bicubic function $S(x, y)$ on $D=[a, b] \times[c, d]$ such that

$$
S\left(x_{i}, y_{j}\right)=F_{i, j}, \quad S(x, y)>0 \text { for all }(x, y) \in D
$$

The $C^{1}$ rational trigonometric bicubic function $S(x, y)$ is simplified to the following form

$$
\begin{aligned}
& S(x, y) \\
& \quad=\frac{a_{i, j}(1-\sin (\theta))^{3}+b_{i, j} \sin (\theta)(1-\sin (\theta))^{2}+c_{i, j} \cos (\theta)(1-\cos (\theta))^{2}+d_{i, j}(1-\cos (\theta))^{3}}{\alpha_{i}(1-\sin (\theta))^{3}+\beta_{i} \sin (\theta)(1-\sin (\theta))^{2}+\gamma_{i} \cos (\theta)(1-\cos (\theta))^{2}+\delta_{i}(1-\cos (\theta))^{3}},
\end{aligned}
$$

with

$$
\begin{aligned}
& a_{i, j}=\frac{(1-\sin (\theta))^{3}}{q_{j}(\varphi)}\left\{(1-\sin (\varphi))^{3} A_{0}+\sin (\varphi)(1-\sin (\varphi))^{2} A_{1}\right. \\
& \left.+\cos (\varphi)(1-\cos (\varphi))^{2} A_{2}+(1-\cos (\varphi))^{3} A_{3}\right\}, \\
& A_{0}=\alpha_{i} \widehat{\alpha}_{j} F_{i, j}, \quad A_{1}=\alpha_{i} \widehat{\beta}_{j} F_{i, j}+2 h_{j} \alpha_{i} \widehat{\alpha}_{j} F_{i, j}^{y}, \quad A_{2}=\alpha_{i} \widehat{\gamma}_{j} F_{i, j+1}-2 h_{j} \alpha_{i} \widehat{\delta}_{j} F_{i, j+1}^{y}, \\
& A_{3}=\alpha_{i} \widehat{\delta}_{j} F_{i, j+1}, \\
& b_{i, j}=\frac{\sin (\theta)(1-\sin (\theta))^{2}}{q_{j}(\varphi)}\left\{(1-\sin (\varphi))^{3} E_{0}+\sin (\varphi)(1-\sin (\varphi))^{2} E_{1}\right. \\
& \left.+\cos (\varphi)(1-\cos (\varphi))^{2} E_{2}+(1-\cos (\varphi))^{3} E_{3}\right\}, \\
& E_{0}=\beta_{i} \widehat{\alpha}_{j} F_{i, j}+2 h_{i} \alpha_{i} \widehat{\alpha}_{j} F_{i, j}^{x} \\
& E_{1}=\beta_{i} \widehat{\beta}_{j} F_{i, j+1}+2 h_{i} \alpha_{i} \widehat{\beta}_{j} F_{i, j}^{x}+2 h_{j}\left(\beta_{i} \widehat{\delta}_{j} F_{i, j}^{y}+2 h_{i} \alpha_{i} \widehat{\alpha}_{j} F_{i, j}^{x y}\right), \\
& E_{2}=\beta_{i} \widehat{\gamma}_{j} F_{i, j+1}+2 h_{i} \alpha_{i} \widehat{\gamma}_{j} F_{i, j+1}^{x}-2 h_{j}\left(\beta_{i} \widehat{\delta}_{j} F_{i, j+1}^{y}+2 h_{i} \alpha_{i} \widehat{\delta}_{j} F_{i, j+1}^{x y}\right), \\
& E_{3}=\beta_{i} \widehat{\delta}_{j} F_{i, j+1}+2 h_{i} \alpha_{i} \widehat{\delta}_{j} F_{i, j+1}^{x} \\
& c_{i, j}=\frac{\cos (\theta)(1-\cos (\theta))^{2}}{q_{j}(\varphi)}\left\{(1-\sin (\varphi))^{3} C_{0}+\sin (\varphi)(1-\sin (\varphi))^{2} C_{1}\right. \\
& \left.+\cos (\varphi)(1-\cos (\varphi))^{2} C_{2}+(1-\cos (\varphi))^{3} C_{3}\right\},
\end{aligned}
$$


Journal of Applied Mathematics

$$
\begin{gathered}
C_{0}=\beta_{i} \widehat{\alpha}_{j} F_{i, j}+2 h_{i} \alpha_{i} \widehat{\alpha}_{j} F_{i, j^{\prime}}^{x}, \\
C_{1}=\gamma_{i} \widehat{\beta}_{j} F_{i+1, j+1}-2 h_{i} \delta_{i} \widehat{\beta}_{j} F_{i+1, j+1}^{x}+2 h_{j}\left(\gamma_{i} \widehat{\alpha}_{j} F_{i+1, j}^{y}-2 h_{i} \delta_{i} \widehat{\alpha}_{j} F_{i+1, j}^{x y}\right), \\
C_{2}=\gamma_{i} \widehat{\gamma}_{j} F_{i+1, j+1}-2 h_{i} \delta_{i} \widehat{\gamma}_{j} F_{i+1, j+1}^{x}-2 h_{j}\left(\gamma_{i} \widehat{\delta}_{j} F_{i+1, j+1}^{y}-2 h_{i} \delta_{i} \widehat{\delta}_{j} F_{i+1, j+1}^{x y}\right), \\
C_{3}=\gamma_{i} \widehat{\delta}_{j} F_{i+1, j+1}^{y}-2 h_{i} \delta_{i} \widehat{\delta}_{j} F_{i+1, j+1}^{x y}, \\
d_{i, j}=\frac{(1-\cos (\theta))^{3}}{q_{j}(\varphi)}\left\{(1-\sin (\varphi))^{3} D_{0}+\sin (\varphi)(1-\sin (\varphi))^{2} D_{1}\right. \\
\left.+\cos (\varphi)(1-\cos (\varphi))^{2} D_{2}+(1-\cos (\varphi))^{3} D_{3}\right\}, \\
D_{0}=\delta_{i} \widehat{\alpha}_{j} F_{i+1, j}, \quad D_{1}=\delta_{i} \widehat{\beta}_{j} F_{i+1, j}+2 h_{j} \delta_{i} \widehat{\alpha}_{j} F_{i+1, j}^{y} \\
D_{2}=\delta_{i} \widehat{\gamma}_{j} F_{i+1, j+1}-2 h_{j} \delta_{i} \widehat{\delta}_{j} F_{i+1, j+1}^{y}, \quad D_{3}=\delta_{i} \widehat{\delta}_{j} F_{i+1, j+1} .
\end{gathered}
$$

Since $\alpha_{i}, \beta_{i}, \gamma_{i}, \delta_{i}, \widehat{\alpha}_{j}, \widehat{\beta}_{j}, \widehat{\gamma}_{j}, \widehat{\delta}_{j}$ are assumed positive real numbers and $0 \leq \theta \leq \pi / 2$, $0 \leq \varphi \leq \pi / 2$, so $S(x, y)>0$ if

$$
a_{i, j}>0, \quad b_{i, j}>0, \quad c_{i, j}>0, \quad d_{i, j}>0 .
$$

$a_{i, j}>0$ if

$$
A_{i}>0, \quad i=0,1,2,3 \text {. }
$$

$A_{i}>0, i=0,1,2,3$, if

$$
\widehat{\beta}_{j}>\frac{-2 h_{j} \widehat{\alpha}_{j} F_{i, j}^{y}}{\pi F_{i, j}}, \quad \widehat{\gamma}_{j}>\frac{2 h_{j} \widehat{\delta}_{j} F_{i, j+1}^{y}}{\pi F_{i, j+1}} .
$$

Similarly, $b_{i, j}>0$ if

$$
E_{i}>0, \quad i=0,1,2,3 .
$$

$E_{i}>0, i=0,1,2,3$, if

$$
\widehat{\gamma}_{j}>\frac{2 h_{j} \widehat{\delta}_{j} F_{i, j+1}^{y}}{\pi F_{i, j+1}},
$$

$\beta_{i}>\operatorname{Max}\left\{C d_{0}, C d_{1}, C d_{2}, C d_{3}, C d_{4}\right\}$, 
with

$$
\begin{gathered}
C d_{0}=\frac{-2 h_{j} \widehat{\alpha}_{j} F_{i, j}^{y}}{\pi F_{i, j}}, \quad C d_{1}=\frac{-2 h_{i} \alpha_{i} F_{i, j}^{x}}{\pi F_{i, j}}, \quad C d_{2}=\frac{-2 h_{i} \alpha_{i} F_{i, j+1}^{x}}{\pi F_{i, j+1}}, \\
C d_{3}=\frac{-2 h_{i} \alpha_{i}\left(\pi \widehat{\beta}_{j} F_{i, j}^{x}+2 h_{j} \widehat{\alpha}_{j} F_{i, j}^{x y}\right)}{\pi^{2} F_{i, j} \widehat{\beta}_{j}+2 \pi h_{j} \widehat{\alpha}_{j} F_{i, j}^{y}}, \quad C d_{4}=\frac{-2 h_{i} \alpha_{i}\left(\pi \widehat{\gamma}_{j} F_{i, j+1}^{x}-2 h_{j} \widehat{\delta}_{j} F_{i, j+1}^{x y}\right)}{\pi^{2} F_{i, j+1} \widehat{\gamma}_{j}-2 \pi h_{j} \widehat{\delta}_{j} F_{i, j+1}^{y}} .
\end{gathered}
$$

Similarly, $c_{i, j}>0$, implies the following constraints:

$$
C_{i}>0, \quad i=0,1,2,3 \text {. }
$$

$C_{i}>0, i=0,1,2,3$, if

$$
\begin{gathered}
\widehat{\beta}_{j}>\frac{-2 h_{j} \widehat{\alpha}_{j} F_{i+1, j}^{y}}{\pi F_{i+1, j}}, \\
\gamma_{i}>\operatorname{Max}\left\{C d_{5}, C d_{6}, C d_{7}, C d_{8}, C d_{9}\right\},
\end{gathered}
$$

where

$$
\begin{gathered}
C d_{5}=\frac{2 h_{j} \widehat{\delta}_{j} F_{i+1, j+1}^{y}}{\pi F_{i+1, j+1}}, \quad C d_{6}=\frac{-2 h_{i} \delta_{i} F_{i+1, j}^{x}}{\pi F_{i+1, j}}, \quad C d_{7}=\frac{2 h_{i} \delta_{i} F_{i+1, j+1}^{x}}{\pi F_{i+1, j+1}}, \\
C d_{8}=\frac{2 h_{i} \delta_{i}\left(\pi \widehat{\beta}_{j} F_{i+1, j}^{x}+2 h_{j} \widehat{\alpha}_{j} F_{i+1, j}^{x y}\right)}{\pi^{2} F_{i+1, j} \widehat{\beta}_{j}+2 \pi h_{j} \widehat{\alpha}_{j} F_{i+1, j}^{y}}, \quad C d_{9}=\frac{2 h_{i} \delta_{i}\left(\pi \widehat{\gamma}_{j} F_{i+1, j+1}^{x}-2 h_{j} \widehat{\delta}_{j} F_{i+1, j+1}^{x y}\right)}{\pi^{2} F_{i+1, j+1} \widehat{\gamma}_{j}-2 \pi h_{j} \widehat{\delta}_{j} F_{i+1, j+1}^{y}},
\end{gathered}
$$

and finally, $d_{i, j}>0$ if

$$
D_{i}>0, \quad i=0,1,2,3 \text {. }
$$

$D_{i}>0, i=0,1,2,3$, if

$$
\widehat{\beta}_{j}>\frac{-2 \widehat{\alpha}_{j} h_{j} F_{i+1, j}^{y}}{\pi F_{i+1, j}}, \quad \widehat{\gamma}_{j}>\frac{2 \widehat{\delta}_{j} h_{j} F_{i+1, j+1}^{y}}{\pi F_{i+1, j+1}} .
$$

The above conditions can be summarized as follows. 
Theorem 5.2. The $C^{1}$ piecewise rational trigonometric bicubic interpolant $S(x, y)$ defined over the rectangular mesh $D=\left[x_{0}, x_{n}\right] \times\left[y_{0}, y_{m}\right]$, in (5.8), is positive if the following sufficient conditions are satisfied

$$
\begin{gathered}
S\left(x_{i}, y_{j}\right)=F_{i, j,} \quad i=0,1,2, \ldots, m ; j=0,1,2 \ldots, n, \\
\alpha_{i}>0, \quad \widehat{\alpha}_{j}>0, \quad \delta_{i}>0, \quad \widehat{\delta}_{j}>0, \\
\widehat{\beta}_{j}>\operatorname{Max}\left\{0, \frac{-2 h_{j} \widehat{\alpha}_{j} F_{i, j}^{y}}{\pi F_{i, j}}, \frac{-2 h_{j} \widehat{\alpha}_{j} F_{i+1, j}^{y}}{\pi F_{i+1, j}}\right\}, \\
\widehat{\gamma}_{j}>\operatorname{Max}\left\{0, \frac{2 h_{j} \widehat{\delta}_{j} F_{i, j+1}^{y}}{\pi F_{i, j+1}}, \frac{2 h_{j} \widehat{\delta}_{j} F_{i+1, j+1}^{y}}{\pi F_{i+1, j+1}}\right\}, \\
\beta_{i}>\operatorname{Max}\left\{0, C d_{0}, C d_{1}, C d_{2}, C d_{3}, C d_{4}\right\}, \\
\gamma_{i}>\operatorname{Max}\left\{0, C d_{5}, C d_{6}, C d_{7}, C d_{8}, C d_{9}\right\},
\end{gathered}
$$

where $C d_{0}, C d_{1}, C d_{2}, C d_{3}, C d_{4}, C d_{5}, C d_{6}, C d_{7}, C d_{8}$, and $C d_{9}$ have been defined in Section 5.

Proof. By combining (5.12)-(5.20), Theorem 5.2 can be easily obtained.

\section{Numerical Examples}

This section exemplifies the curve and surface scheme for positive data developed in Sections 4 and 5. The $C^{1}$ rational trigonometric cubic function (2.1) is first used to interpolate the positive data sets taken in Tables 1 and 2, respectively. Arbitrary values are assigned to free parameters and resulting curves are shown in Figures 1 and 3, respectively. It is clear from Figures 1 and 3 that rational trigonometric cubic function does not preserve the shape of data for arbitrary values of free parameters. The positive curves for the same data sets are produced in Figures 2 and 4 by the rational positivity preserving surface scheme developed in Section 5.1.

The 3D positive data set in Tables 3 and 4 are generated from the functions $F(x, y)=$ $x^{2}+x y+y^{2}+0.1$ and $F(x, y)=x^{2}+y^{2}$, respectively.

Figure 5 and Figure 7 are produced by interpolating the positive data sets in Tables 3 and 4 , respectively by $C^{1}$ rational trigonometric bicubic function for arbitrary values of free parameter. Positive surfaces in Figures 6 and 8 are produced by interpolating the same data by the positivity preserving scheme developed in Section 5.2.

\section{Conclusion}

In recent years, the trigonometric curves are attaining considerable importance due to their trigonometric basis functions which provide them the opportunity to construct conics, cylinders, surface of revolution, catenary, and so on. Han [5] used B-spline representation of cubic trigonometric curve with shape parameter for shape designing. In this paper, a Hermite form of $C^{1}$ rational trigonometric function is introduced. The developed interpolant 


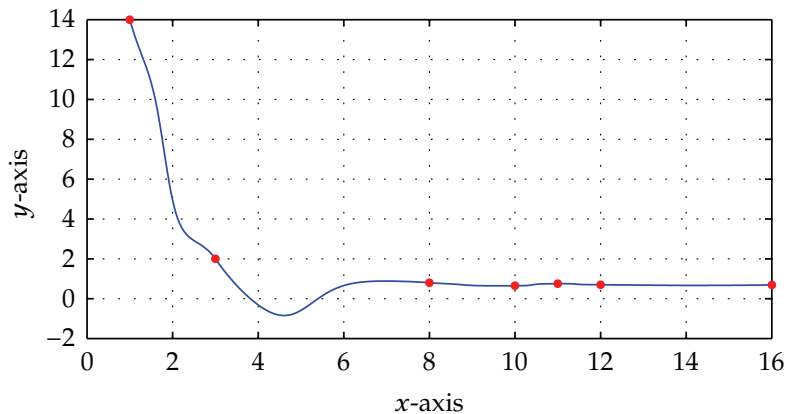

Figure 1: $C^{1}$ rational trigonometric cubic function with $\alpha_{i}=0.5, \beta_{i}=1.0, \gamma_{i}=0.5$, and $\delta_{i}=1.0$.

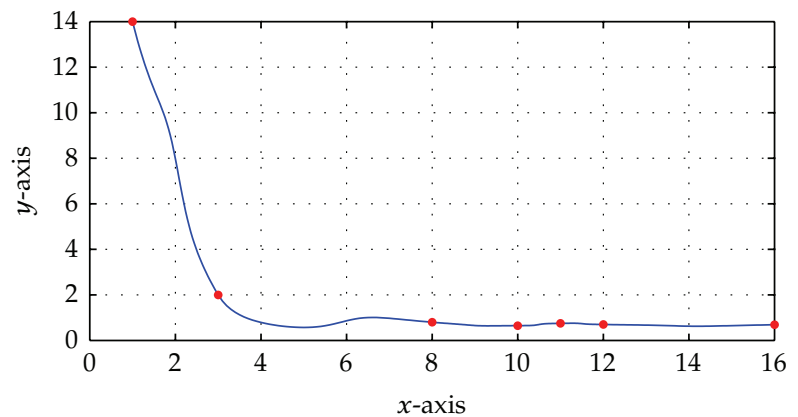

Figure 2: $C^{1}$ positive rational trigonometric cubic function with $\alpha_{i}=0.5, \delta_{i}=1.0$.

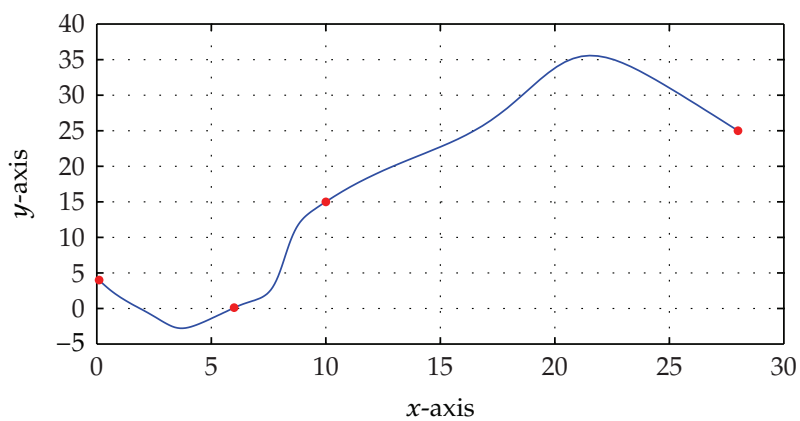

Figure 3: $C^{1}$ rational trigonometric cubic function with $\alpha_{i}=0.5, \beta_{i}=1.0, \gamma_{i}=0.5$, and $\delta_{i}=1.0$.

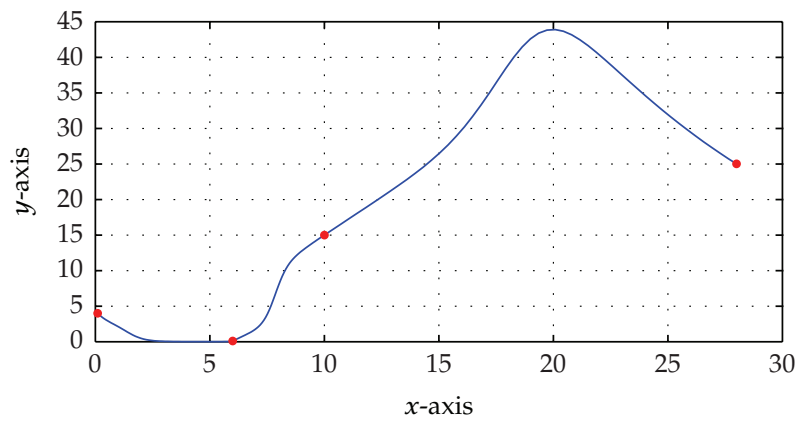

Figure 4: $C^{1}$ rational trigonometric cubic function with $\alpha_{i}=0.5, \delta_{i}=1.0$. 


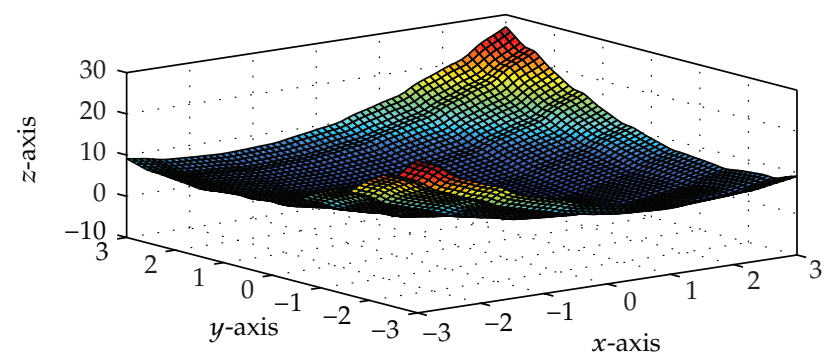

Figure 5: $C^{1}$ rational trigonometric bicubic function with $\alpha_{i}=8, \beta_{i}=8, \gamma_{i}=8, \delta_{i}=8, \widehat{\alpha}_{j}=4, \widehat{\beta}_{j}=4, \widehat{\gamma}_{j}=$ 4 , and $\widehat{\delta}_{j}=4$.

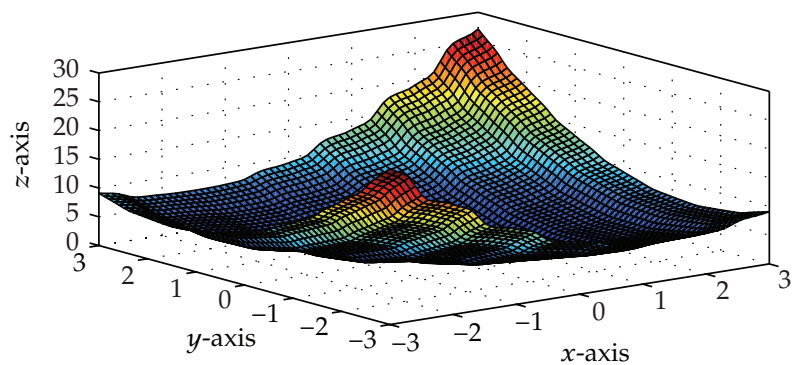

Figure 6: $C^{1}$ positive rational trigonometric bicubic function with $\alpha_{i}=8, \delta_{i}=8, \widehat{\alpha}_{j}=8$, and $\widehat{\delta}_{j}=8$.

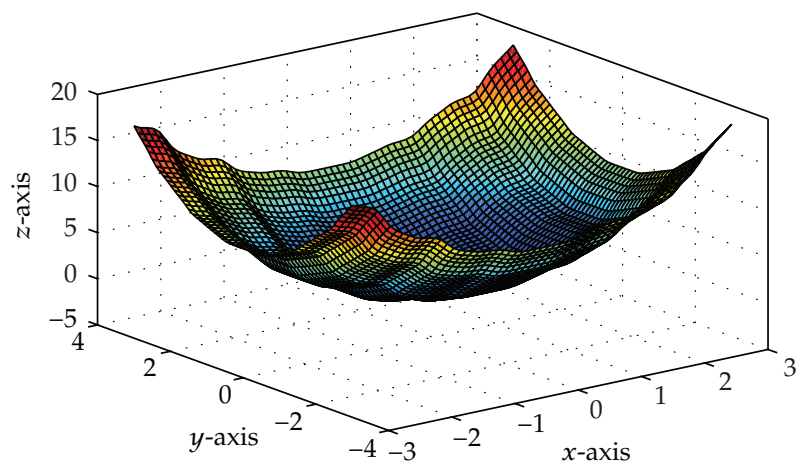

Figure 7: $C^{1}$ rational trigonometric bicubic function with $\alpha_{i}=7, \beta_{i}=7, \gamma_{i}=7, \delta_{i}=7, \widehat{\alpha}_{j}=3, \widehat{\beta}_{j}=3, \widehat{\gamma}_{j}=$ 3 , and $\widehat{\delta}_{j}=3$.

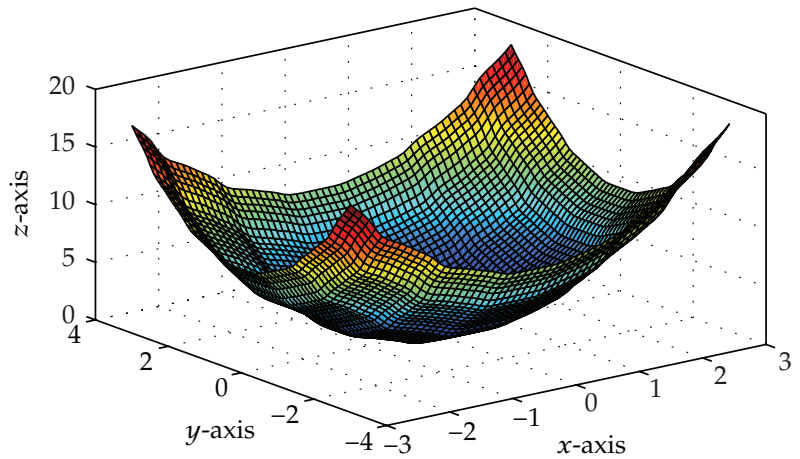

Figure 8: $C^{1}$ positive rational trigonometric bicubic function with $\alpha_{i}=6, \delta_{i}=6, \widehat{\alpha}_{j}=6$, and $\widehat{\delta}_{j}=6$. 
Table 1: A 2D positive data set.

\begin{tabular}{cccccccc}
\hline$x$ & 1 & 3 & 8 & 10 & 11 & 12 & 16 \\
$y$ & 14 & 2 & 0.8 & 0.65 & 0.75 & 0.70 & 0.69 \\
\hline
\end{tabular}

Table 2: A 2D positive data set.

\begin{tabular}{lcccc}
\hline$x$ & 0.1 & 6 & 10 & 28 \\
$y$ & 4 & 0.1 & 15 & 25 \\
\hline
\end{tabular}

Table 3: A 3D positive data set.

\begin{tabular}{lccccccc}
\hline$y$ & -3 & -2 & -1 & 0 & 1 & 2 & 3 \\
\hline-3 & 27.1 & 19.1 & 13.1 & 9.1 & 7.1 & 7.1 & 9.1 \\
-2 & 19.1 & 12.1 & 7.1 & 4.1 & 3.1 & 4.1 & 7.1 \\
-1 & 13.1 & 7.1 & 3.1 & 1.1 & 1.1 & 3.1 & 7.1 \\
0 & 9.1 & 4.1 & 1.1 & 0.1 & 1.1 & 4.1 & 9.1 \\
1 & 7.1 & 3.1 & 1.1 & 1.1 & 3.1 & 7.1 & 13.1 \\
2 & 7.1 & 4.1 & 3.1 & 4.1 & 7.1 & 12.1 & 19.1 \\
3 & 9.1 & 7.1 & 7.1 & 9.1 & 13.1 & 19.1 & 27.1 \\
\hline
\end{tabular}

Table 4: A 3D positive data set.

\begin{tabular}{lccccccc}
\hline$y$ & -3 & -2 & -1 & $x$ & 0 & 1 & 2 \\
\hline-3 & 18 & 13 & 10 & 9 & 10 & 13 & 18 \\
-2 & 13 & 8 & 5 & 4 & 5 & 8 & 13 \\
-1 & 10 & 5 & 2 & 1 & 2 & 5 & 10 \\
0 & 9 & 4 & 1 & 0 & 1 & 4 & 9 \\
1 & 10 & 5 & 2 & 1 & 2 & 5 & 10 \\
2 & 13 & 8 & 5 & 4 & 5 & 8 & 13 \\
3 & 18 & 13 & 10 & 9 & 10 & 13 & 18 \\
\hline
\end{tabular}

involves four free parameters in each subintervals. Constraints are developed on two of these parameters to preserve positive shape of data, while the remaining was free for shape modification. The developed $C^{1}$ interpolant is extended to $C^{1}$ rational trigonometric bicubic interpolant for the treatment positive surface data. It is observed that error of developed interpolant is of order three same as the polynomial interpolant. The surface interpolation scheme developed in this paper is $C^{1}$, whereas in $[8,9]$ it was $C^{0}$ only. Moreover, the $C^{1}$ continuity of curve and surface data interpolant does constrain step length.

\section{References}

[1] M. R. Asim and K. W. Brodlie, "Curve drawing subject to positivity and more general constraints," Computers and Graphics, vol. 27, pp. 469-485, 2003.

[2] S. Butt and K. W. Brodlie, "Preserving positivity using piecewise cubic interpolation," Computers and Graphics, vol. 17, no. 1, pp. 55-64, 1993. 
[3] T. N. T. Goodman, B. H. Ong, and M. L. Sampoli, “Automatic interpolation by fair, shape-preserving space curves," Computer Aided Design, vol. 30, pp. 813-822, 1998.

[4] T. N. T. Goodman, "Shape preserving interpolation by curves," in Algorithms for Approximation, J. Levesley, I. J. Anderson, and J. C. Mason, Eds., pp. 24-35, University of Huddersfeld, 2002.

[5] X. Han, "Cubic trigonometric polynomial curves with a shape parameter," Computer Aided Geometric Design, vol. 21, no. 6, pp. 535-548, 2004.

[6] K. W. Brodlie, P. Mashwama, and S. Butt, "Visualization of surface data to preserve positivity and other simple constraints," Computers and Graphics, vol. 19, no. 4, pp. 585-594, 1995.

[7] Q. Duan, Y. Zhang, and E. H. Twizell, "A bivariate rational interpolation and the properties," Applied Mathematics and Computation, vol. 179, no. 1, pp. 190-199, 2006.

[8] M. Z. Hussain and M. Hussain, "Visualization of data subject to positive constraints," Journal of Information and Computing Science, vol. 1, no. 3, pp. 149-160, 2006.

[9] M. Z. Hussain and M. Sarfraz, "Positivity-preserving interpolation of positive data by rational cubics," Journal of Computational and Applied Mathematics, vol. 218, no. 2, pp. 446-458, 2008.

[10] Q. Duan, H. Zhang, Y. Zhang, and E. H. Twizell, "Error estimation of a kind of rational spline," Journal of Computational and Applied Mathematics, vol. 200, no. 1, pp. 1-11, 2007. 


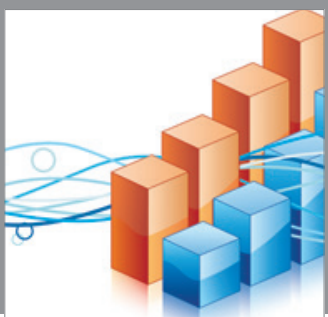

Advances in

Operations Research

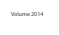

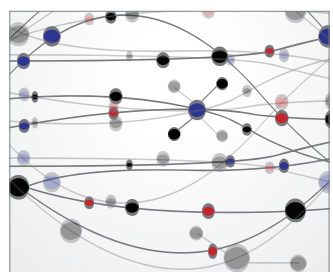

\section{The Scientific} World Journal
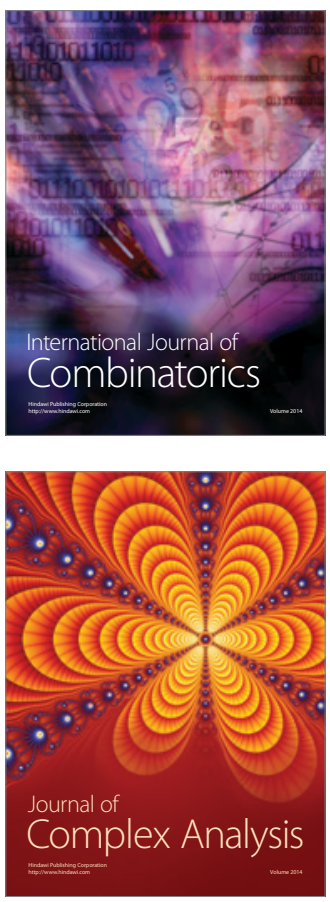

International Journal of

Mathematics and

Mathematical

Sciences
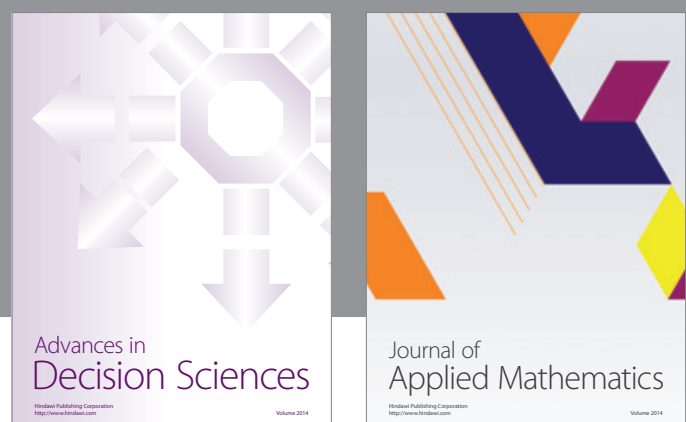

Journal of

Applied Mathematics
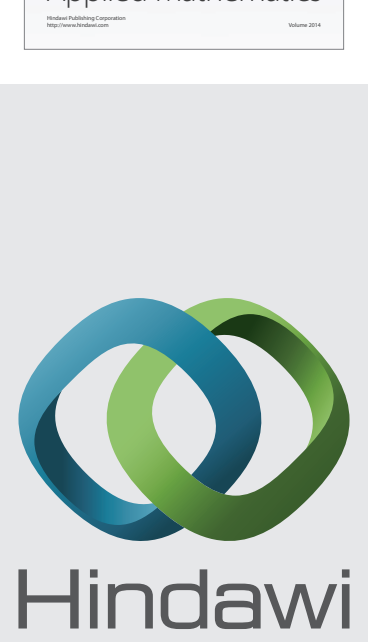

Submit your manuscripts at http://www.hindawi.com
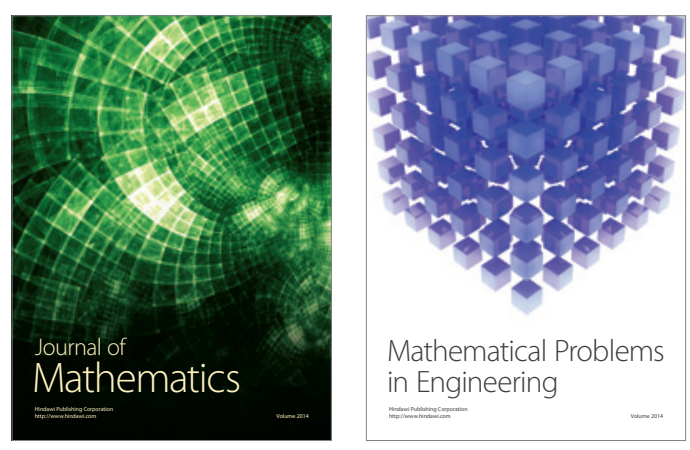

Mathematical Problems in Engineering
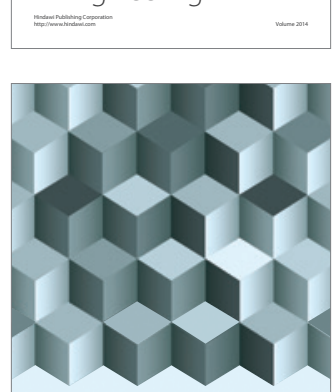

Journal of

Function Spaces
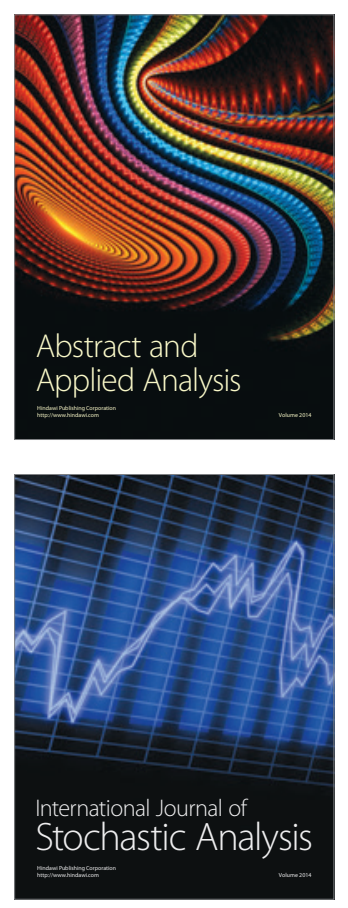

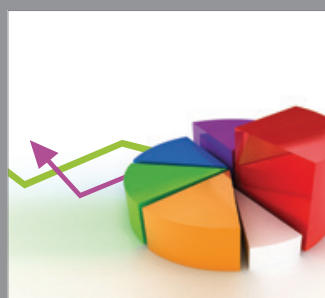

ournal of

Probability and Statistics

Promensencen
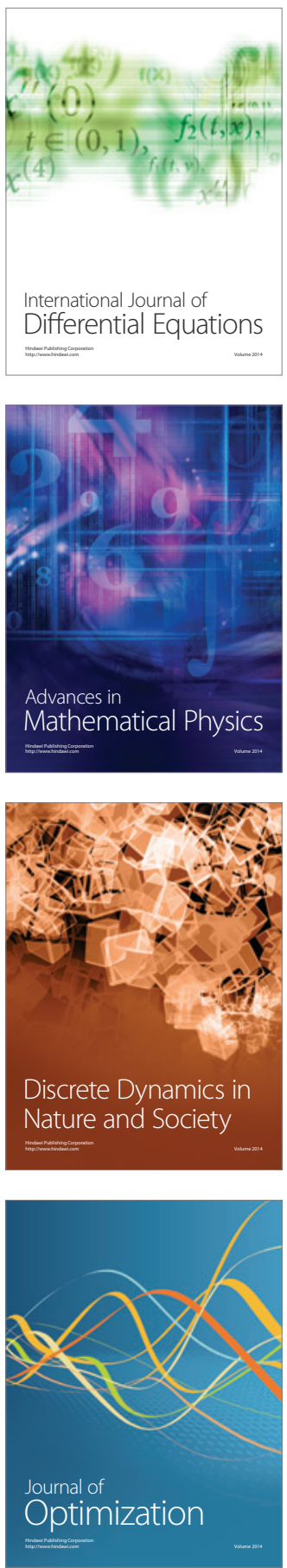